\title{
ON GENERALIZED LORENTZ-ZYGMUND SPACES
}

\author{
B. OPIC AND L. PICK
}

\begin{abstract}
We study generalized Lorentz-Zygmund spaces with broken logarithmic functions. We derive necessary and sufficient conditions for embeddings between them. We give a complete characterization of their associate spaces. We establish necessary and sufficient conditions for a generalized Lorentz-Zygmund space to be a Banach function space and to have absolutely continuous (quasi-)norm. We describe completely relations between these spaces and Orlicz spaces.
\end{abstract}

\section{Mathematics subject classification (1991): 46E30, 26D15.}

Key words and phrases: Generalized Lorentz-Zygmund spaces, broken logarithmic functions, embedding theorems, associate spaces, Banach function spaces, Orlicz spaces, absolutely continuous (quasi-)norms.

\section{REFERENCES}

[BR] C. Bennett And K. Rudnick, On Lorentz-Zygmund spaces, Dissertationes Math. 175 (1980), $1-72$.

[BS] C. Bennett and R. Sharpley, Interpolation of Operators, Pure and Appl. Math., vol. 129, Academic Press, New York, 1988.

[BW] H. BRÉZIS AND S. WAINGER, A note on limiting cases of Sobolev embeddings and convolution inequalities, Comm. Partial Diff. Eq. 5 (1980), 773-789.

[CPSS] M. CARRo, L. Pick, J. Soria AND V.D. Stepanov, On embeddings between classical Lorentz spaces, Preprint Centre de Recerca Barcelona 385 (1998), 1-36, (to appear).

[CS1] M. CARRO AND J. SORIA, Weighted Lorentz spaces and the Hardy operator, J. Funct. Analysis 112 (1993), 480-494.

[CS2] M. CARRO AND J. SORIA, Boundedness of some integral operators, Canad. J. Math. 45 (1993), $1155-1166$.

[CS3] M. CARRO AND J. SORIA, The Hardy-Littlewood maximal function and weighted Lorentz spaces, J. London Math. Soc. 55 (1997), 146-158.

[CP] M. CWIKEL AND E. PUSTYLNIK, Sobolev type embeddings in the limiting case, J. Fourier Anal. Appl. (to appear).

[EGO1] D.E. EDMUNDS, P. GURKA AND B. OPIC, Double exponential integrability of convolution operators in generalized Lorentz-Zygmund spaces, Indiana Univ. Math. J. 44 (1995), 19-43.

[EGO2] D.E. EDMUNDS, P. GURKA AND B. OPIC, Double exponential integrability, Bessel potentials and embedding theorems, Studia Math. 115 (1995), 151-181.

[EGO3] D.E. EDMUNDS, P. GURKA AND B. OPIC, Sharpness of embeddings in logarithmic Bessel-potential spaces, Proc. Roy. Soc. Edinburgh 126A (1996), 995-1009.

[EGO4] D.E. EDMUNDS, P. GURKA AND B. OPIC, On embeddings of logarithmic Bessel potential spaces, J. Functional Anal. 146 (1997), 116-150.

[EGO5] D.E. EDMUNDS, P. GURKA AND B. OpIC, Norms of embeddings of logarithmic Bessel potential spaces, Proc. Amer. Math. Soc. 126 (98), 2417-2425.

[EKP] D.E. Edmunds, R.A. KeRman AND L. PICK, Optimal Sobolev imbeddings involving rearrangement-invariant quasinorms (to appear).

[EO] W.D. EVANS AND B. OPIC, Real interpolation with logarithmic functors and reiteration (to appear).

[EOP1] W.D. EVANS, B. OPIC AND L. PICK, Interpolation of operators on scales of generalized Lorentz-Zygmund spaces, Math. Nachr. 182 (1996), 127-181. 
[EOP2] W.D. Evans, B. OPIC AND L. PICK, Real interpolation with logarithmic functors (to appear).

[GHS] M.L. Gol'dman, H.P. HeINIG AND V.D. STEPANOV, On the principle of duality in Lorentz spaces, Canad. J. Math. 48 (1996), 959-979.

[GM] M.E. GomeZ AND M. MILMAN, Extrapolation spaces and almost-everywhere convergence of singular integrals, J. London Math. Soc. 34 (1986), 305-316.

[GO] P. GURKA AND B. OPIC, Global limiting embeddings of logarithmic Bessel potential spaces, Math. Ineq. Appl. 1 (1998), 565-584.

[H] K. HANSSON, Imbedding theorems of Sobolev type in potential theory, Math. Scand. 45 (1979), $77-102$.

[KR] M.A. Krasnosel'Skit and YA. B. RutitskiI, Convex Functions and Orlicz Spaces, Noordhoff, Groningen, 1961.

[La] S. LAI, Weighted norm inequalities for general operators on monotone functions, Trans. Amer. Math. Soc. 340 (1993), 811-836.

[Lo1] G.G. LoRENTZ, On the theory of spaces $\Lambda$, Pacific J. Math. 1 (1951), 411-429.

[Lo2] G.G. LoRENTZ, Relations between function spaces, Proc. Amer. Math. Soc. 12 (1961), 127-132.

[Lu] W.A.J. LuXEMBURG, Banach Function Spaces, Thesis, Delft, 1955.

[OK] B. OPIC AND A. KUFNER, Hardy-type inequalities, Pitman Research Notes in Math., Series 219, Longman Sci \& Tech., Harlow, 1990.

[OT] B. OPIC AND W. TREBELS, Bessel potentials with logarithmic components and Sobolev-type embeddings (to appear).

[Sa] E.T. SAWYER, Boundedness of classical operators on classical Lorentz spaces, Studia Math. 96 (1990), 145-158.

[Sh] R. Sharpley, Spaces $\Lambda_{\alpha}(X)$ and interpolation, J. Funct. Anal. 11 (1972), 479-513.

[So] J. SORIA, Lorentz spaces of weak-type, Quart. J. Math. Oxford 49 (1998), 93-103.

[St] V.D. STEPANOV, The weighted Hardy's inequality for nonincreasing functions, Trans. Amer. Math. Soc. 338 (1993), 173-186. 